\title{
R-Peak Detection in Holter ECG Signals Using Non-Negative Matrix Factorization
}

\author{
Pauline Guyot ${ }^{1,3}$, Pascal Voiriot ${ }^{4}$, El-Hadi Djermoune ${ }^{1}$, Stéphane Papelier ${ }^{4}$, \\ Céline Lessard ${ }^{4}$, Mathieu Felices ${ }^{5}$, Thierry Bastogne $e^{1,2,3}$ \\ ${ }^{1}$ CRAN, Université de Lorraine, CNRS UMR 7039, Vandouvre-lès-Nancy, France \\ ${ }^{2}$ INRIA, BIGS, Vandœuvre-lès-Nancy, France \\ ${ }^{3}$ CYBERnano, Villers-lès-Nancy, France \\ ${ }^{4}$ Banook Group, Nancy, France \\ ${ }^{5}$ PhinC Development, Massy, France
}

\begin{abstract}
Holter monitoring is mainly used for medical followup and diagnosis of patients with suspected cardiac arrhythmia such as heart rhythm irregularities that can be missed during classical electrocardiogram recording (ECG). However, these long-term continuous recordings represent a large amount of data that cannot be processed by hand. In this article, we present a new method based on Non-negative Matrix Factorization (NMF) to detect $R$ peaks in Holter signals. The approach consists in two stages: source separation based on the different timefrequency patterns of the QRS complexes and the other waves of the signal ( $P$ and $T$ waves) and R-peak detection using Automatic Objective Thresholding (AOT). The proposed approach is validated on the MIT-BIH Arrhythmia database and achieves an average sensitivity of $99.59 \%$ and a precision of $99.69 \%$. Using the MIT-BIH Noise Stress Test database, we also show the ability of our approach to discriminate R-peaks in signals contaminated with different noises.
\end{abstract}

\section{Introduction}

In recent years, there has been an increasing interest in Holter recordings as it allows reliable detection of cardiac pathologies with infrequent short-term transient symptoms. Yet, one of the key issues concerning longduration Holter electrocardiograms (ECGs) is the amount of data to be processed by the clinician. The development of an automatic tool able to delineate and detect ECG events has become essential. One of the main parameters representing the heart health of the patient is the heart rate variation as it provides great knowledge about cardiovascular activity. It is computed by detecting all contractions of the heart, i.e. all $\mathrm{R}$ peaks.
To this aim, various methods based on wavelet transform [1], Hilbert transform [2], Bayesian framework [3] or Pan-Tompkins algorithm [4] have been proposed. The focus on this paper is to demonstrate the feasibility of source separation in ECG signals in order to detect $\mathrm{R}$ peaks, using a blind source separation algorithm called Non-negative Matrix Factorization (NMF). Some researches have been made combining electrocardiogram and NMF, such as ECG-EMG separation [5] or extraction of fetal ECG [6], but to the best of our knowledge, none on the separation of the ECG waves.

\section{Methodology}

\subsection{Non-Negative Matrix Factorization}

Non-negative Matrix Factorization is an unsupervised learning method which aims at decomposing a nonnegative matrix $\left(V \in \Re^{M \times N}\right.$ ) into two non-negative matrices $\left(W \in \Re^{M \times S}\right.$ and $H \in \Re^{S \times N}$ ):

$$
V=W H^{T}=\sum_{s=1}^{S} w_{s} h_{s}^{T}
$$

where $(\cdot)^{T}$ stands for matrix transposition and $S$ is the number of sources to decompose in the input signals $V$ (consisting in $M$ samples of $N$ non-negative variables). $W$ is a basis matrix, meaning that each column $w_{s}$ of $W$ is a basis vector (source) of the input mixture $V$ and $H$ is the coefficient matrix, each row $h_{s}^{T}$ of $H$ representing the activation set for each point of $V$. The matrices $W$ and $H$ are computed iteratively in order to minimize the distance between $V$ and $W H^{T}$ through the following process:

$$
W_{i j} \leftarrow W_{i j} \frac{\left(V H^{T}\right)_{i j}}{\left(W H H^{T}\right)_{i j}}
$$




$$
H_{i j} \leftarrow H_{i j} \frac{\left(W^{T} V\right)_{i j}}{\left(W^{T} W H\right)_{i j}}
$$

The minimization of the error relies on a cost function defined by:

$$
D\left(V \mid W H^{T}\right)=\sum_{i} \sum_{j} d\left(V_{i j} \mid\left(W H^{T}\right)_{i j}\right) .
$$

One can use for $d$ the squared Euclidian distance, the generalized Kullback-Leibler divergence or the Itakura-Saito divergence [7]. It is also possible to add optional constraints to enforce some desired properties to the matrices $W$ and $H$.

\subsection{Proposed detection technique}

\subsubsection{Sources}

NMF is a technique solving source separation problems. Therefore, our goal was to test this method on Holter signals to separate the different waves. The first intuition would be to make a medical separation between the atrial and the ventricular activity (P wave v.s. QRS complex and $\mathrm{T}$ wave) as they produce distinct temporal patterns. However, as $\mathrm{P}$ and $\mathrm{T}$ waves have very similar frequency patterns, it would be very difficult to separate them for NMF. The easiest decomposition in terms of signal processing would be QRS complexes v.s. non-QRS parts (including $\mathrm{P}$ wave, $\mathrm{T}$ wave and potentially $\mathrm{U}$ wave).

In [8], the typical relative power spectrum of an ECG signal is presented with its different components: (1) QRS complex is concentrated in the mid-frequency range, with its fundamental frequency at $10 \mathrm{~Hz}$. R peak with its fast slope is a high-frequency component around $100 \mathrm{~Hz}$; (2) $\mathrm{P}$ and $\mathrm{T}$ waves have a low frequency distribution (around 1-2 Hz); (3) Noise can be low-frequency (motion artifact and baseline wander), mid-frequency (muscle noise) or frequency-specific (as power grid, 50-60 Hz).

The main difficulty comes from the fact that QRS complexes and non-QRS parts ( $\mathrm{P}$ and $\mathrm{T}$ waves) can overlap in the frequency domain.

\subsubsection{Step 1: NMF}

Our method consists in two stages, namely source separation and detection of $\mathrm{R}$ peaks. A pre-processing is not necessary but we filtered the ECG signal in order to remove power line interference $(50-60 \mathrm{~Hz})$. No baseline wander removal algorithm is needed in this approach.

The magnitude of the spectrogram of the ECG signal is chosen to meet the non-negativity requirement ( $V$ matrix). The NMF algorithm is applied with $S=2$ sources without constraints and we chose a random initialization.

The separation gives two sources as outputs: the first source is generally the one with most energy which represents QRS complexes and the second one contains the non-QRS parts. Noise can be put in either sources depending on its frequency distribution.

\subsubsection{Step 2: Peak detection using Auto- matic Objective Thresholding (AOT)}

The QRS source given in output of the NMF algorithm takes the form of spike activity. Therefore, the method used to detect R-peaks is inspired by the work of Tanskanen et al. [9] proposed to detect neuronal spikes. Classical detection algorithm for R-peaks (Pan-Tompkins, Wavelet...) were not sufficiently reliable in our case.

We modified some parameters of the AOT method into fitting the ECG signal. The histogram of the first minute of the signal is computed with $k=500$ bins. The smoothed gradient of the histogram is then calculated and the central pattern is delineated in order to get the two threshold levels above and below with which spikes will be detected. We applied a default refractory period of 0.3 second.

\section{Results and discussion}

\subsection{Dataset}

The MIT-BIH Arrhythmia database (MITADB) was used to test our method. It contains 48 half-hour Holter ECG recordings, of two channels each. The recordings are sampled at $360 \mathrm{~Hz}$ with 11-bit resolution over a 10 $\mathrm{mV}$ range. Two or more cardiologists independently annotated each record and disagreements were resolved to obtain reference annotations for each beat. The ECG records have acceptable quality, sharp and tall $\mathrm{P}$ and $\mathrm{T}$ waves, negative $\mathrm{R}$ waves, small $\mathrm{R}$-peak amplitudes, wider $\mathrm{R}$ waves, muscle noise, baseline drift, sudden changes in heartbeat morphology, multiform PVC, long pauses, and irregular heart rhythms [10]. In order to better compare with other methods, we worked on the first channel of each ECG recording and excluded episodes of ventricular flutter from record 207. We also used the complex baseline wander, electrode artifacts and motion artifacts from the MIT-BIH Noise Stress Test Database (NSTDB) to test the noise tolerance of our method. In our second test, we contaminated all signals from the MITADB with those three types of artifacts to create noisy ECG signals.

\subsection{Evaluation parameters}

We computed three quantitative indicators: True Positives (TP) when an R peak is correctly detected by our algorithm, False Positives (FP) when noise is detected as an $R$ peak and False Negatives (FN) when an R peak is missed. Then, the following parameters are used for the 

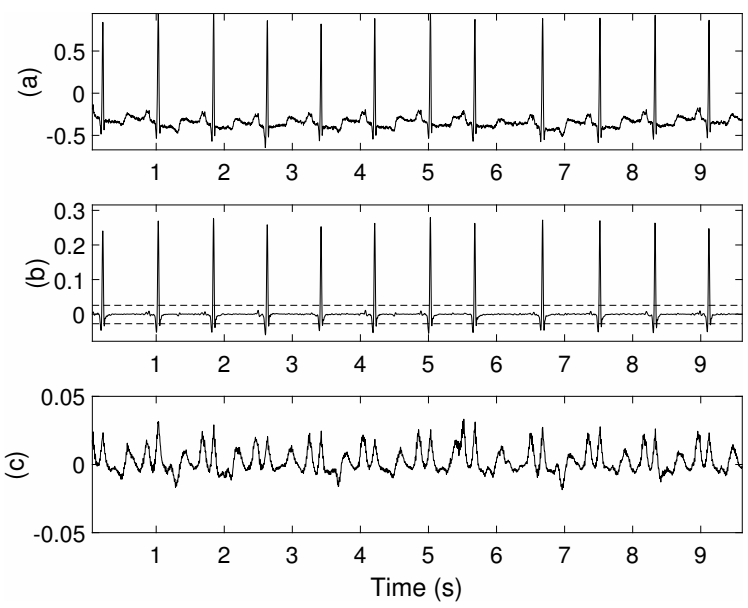

Figure 1. Source separation of record $\mathbf{1 0 0}$ from MITADB with Non-Negative Matrix Factorization (NMF). (a) Original ECG, (b) First extracted source, representing QRS complexes (c) Second source, representing $\mathrm{P}$ and $\mathrm{T}$ waves and some remaining components of QRS complexes.

evaluation of our algorithm: Sensitivity $\left(\mathrm{Se}=\frac{\mathrm{TP}}{\mathrm{TP}+\mathrm{FN}}\right.$. $100 \%)$, Precision $\left(\mathrm{Pr}=\frac{\mathrm{TP}}{\mathrm{TP}+\mathrm{FP}} \cdot 100 \%\right)$ and Accuracy $\left(\mathrm{Acc}=\frac{\mathrm{FP}}{\mathrm{TP}+\mathrm{FP}+\mathrm{FN}} \cdot 100 \%\right)$.

\subsection{Experiment results}

Complete separation results are shown in Figure 1 for Record 100 of the MITADB. Using NMF source separation algorithm on Holter recordings has several advantages. It firstly allows to clearly isolate QRS complexes even if some QRS components remain in the second source as QRS complexes partially overlap P and T waves in the frequency domain (see Figure 1). Variation dynamics between QRS complexes and the other waves are sufficient to separate the predicted sources. In Figure $2 b$ and Figure $3 \mathrm{~b}$, separation is also represented in the case of baseline wander and an abnormal ventricular activity. The separation achieves good results for Figure 1 and Figure 2: in the first source, R-peaks have a spike-like morphology and the baseline wander is removed. However in Figure 3, the QRS separation is less efficient: R-peaks are still detectable in the QRS source but some arrhythmic beats (ventricular couplets) have a very low amplitude.

Our method uses a constraint-free version of the NMF algorithm, and one can easily imagine to add constraints in order to better separate the two sources. In our case, the actual separation is sufficient to detect R-peaks in most cases. However, if QRS onsets and offsets have to be determined as well, our separation will probably not be accurate enough.

NMF algorithm removes any baseline wander in its QRS output source. The case of higher frequency noise is more
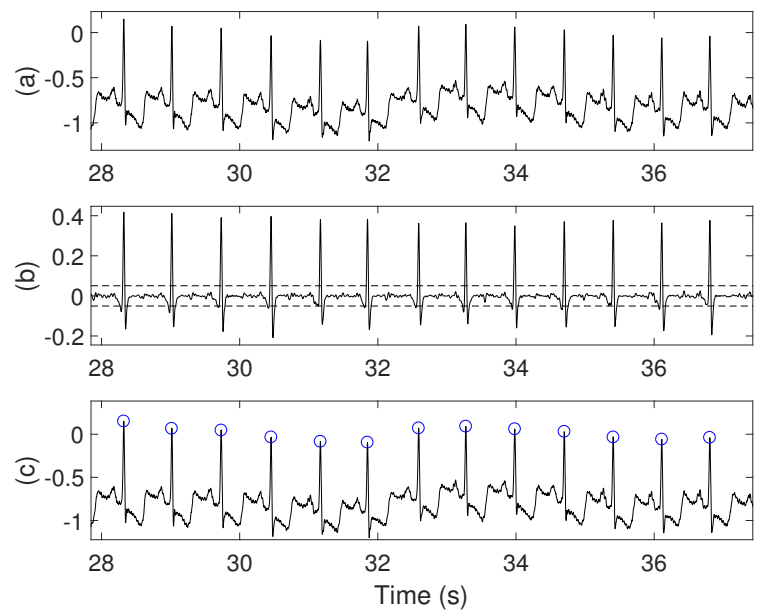

Figure 2. R-peak detection of record $\mathbf{1 1 2}$ from MITADB, using Automatic Objective Thresholding (AOT). (a) Original ECG, (b) QRS source with the threshold levels (c) Original ECG annotated with detected $\mathrm{R}$ peaks (circles).

complicated: it can be put in any of the two sources according to its frequency composition. If it is very low frequency, it will go in the non-QRS parts (less than $10 \mathrm{~Hz}$, as baseline wander) and if higher, it will be found in the first source.

Two examples of R peak detection using AOT are presented in Figure 2 and Figure 3. In Table 1, results of our method are compared with references. The algorithm has a total detection failure of 863 beats, with 325 FP beats and 538 FN beats, out of 109,809 beats. The sensitivity, which measures the ability to detect true beats, is slightly less than the other methods ( $99.59 \%$ ) because the thresholding levels computed on the first minute of the signal are applied on the whole ECG. Also, as the QRS source is not polluted by baseline wander or tall $\mathrm{P}$ and $\mathrm{T}$ waves, a more sensitive thresholding can be created to be more robust to cases like in Figure 3. The precision of our algorithm, indicator of its ability to discriminate false and true beats, is similar to the other methods. The accuracy of our algorithm is also slightly lower than the other methods due its sensitivity.

In a second analysis, we polluted the signals from the MITADB with the different noises provided in NSTDB. Results are found in Table 1 for baseline wander, electrode motion and muscle artifacts. Because our method has not been re-tuned to fit the new signals, the results are promising and show that the algorithm can detect R-peaks with different noises.

\section{Conclusion}

In this paper, a new strategy to detect R-peaks in Holter recordings is presented. This algorithm separates QRS 

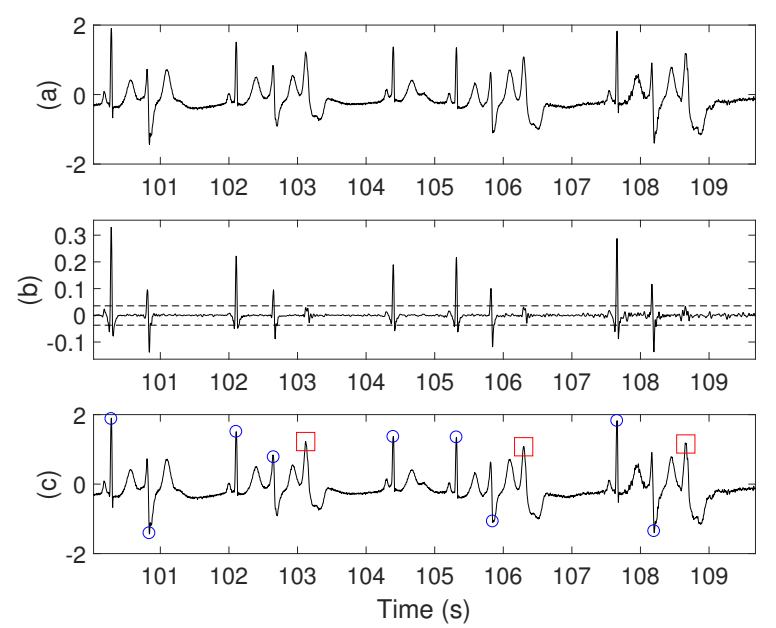

Figure 3. R-peak detection of record $\mathbf{1 0 6}$ from MITADB, using Automatic Objective Thresholding (AOT). (a) Original ECG, (b) QRS source with the threshold levels (c) is the original ECG annotated with detected R peaks (circles) and missed ones (squares).

\begin{tabular}{|l|cccc|}
\hline Method & Beats & Se (\%) & Pr (\%) & Acc (\%) \\
\hline Pan \& Tompkins [4] & 116137 & 99.76 & 99.56 & 99.33 \\
Christov [11] & 110050 & 99.74 & 99.65 & 99.56 \\
Zidelmal et al [12] & 109494 & 99.64 & 99.82 & 99.47 \\
Chouakri et al [13] & 110934 & 98.68 & 97.24 & 96.03 \\
\hline Our algorithm (1) & 109809 & 99.59 & 99.69 & 99.22 \\
Our algorithm (2) & 109809 & 94.76 & 96.52 & 91.63 \\
Our algorithm (3) & 109809 & 90.21 & 84.87 & 77.71 \\
Our algorithm (4) & 109809 & 96.70 & 89.23 & 86.60 \\
\hline
\end{tabular}

Table 1. Comparison of R-peak detection indicators obtained on the MIT-BIH and NSTDT databases for several methods. (1) Original MIT-BIH Arrhythmia Database (2) MITADB with baseline wander (3) MITADB with electrode motion artifacts (4) MITADB with muscle artifacts.

complexes from non-QRS parts ( $\mathrm{P}$ and $\mathrm{T}$ waves) using Non-negative Matrix Factorization, a source separator. In our experiments on the MIT-BIH Arrhythmia database, our method achieves comparable results in terms of sensitivity, precision and accuracy with other existing methods and proves to be noise-tolerant. Future works will focus on the use of the second source in the detection of atrial fibrillation.

\section{References}

[1] G. de Lannoy, B. Frenay, M. Verleysen, and J. Delbeke, Supervised ECG delineation using the wavelet transform and hidden Markov models, SpringerLink, pp. 2225, 2009.

[2] D. Benitez, P. A. Gaydecki, A. Zaidi, and A. P. Fitzpatrick, The use of the Hilbert transform in ECG signal analysis, Computers in Biology and Medicine, vol. 31, no. 5, pp. 399406, 2001.
[3] O. Sayadi and M. B. Shamsollahi, A model-based Bayesian framework for ECG beat segmentation, Physiol. Meas., vol. 30, no. 3, p. 335, 2009.

[4] J. Pan and W. J. Tompkins, A Real-Time QRS Detection Algorithm, IEEE Transactions on Biomedical Engineering, vol. BME-32, no. 3, pp. 230236, Mar. 1985.

[5] M. Niegowski and M. Zivanovic, ECG-EMG separation by using enhanced non-negative matrix factorization, in 2014 36th Annual International Conference of the IEEE Engineering in Medicine and Biology Society, 2014, pp. 42124215

[6] P. J. He, X. M. Chen, Y. Liang, and H. Z. Zeng, Extraction for fetal ECG using single channel blind source separation algorithm based on multi-algorithm fusion, MATEC Web of Conferences, vol. 44, p. 01026.

[7] F. ITAKURA, Analysis synthesis telephony based on the maximum likelihood method, International Congress on Acoustics, 1968, pp. 1720, 1968.

[8] N. V. Thakor, From Holter monitors to automatic defibrillators: developments in ambulatory arrhythmia monitoring, IEEE Transactions on Biomedical Engineering, vol. BME31, no. 12, pp. 770778, Dec. 1984.

[9] J. M. A. Tanskanen, F. E. Kapucu, I. Vornanen, and J. A. K. Hyttinen, Automatic objective thresholding to detect neuronal action potentials, in 2016 24th European Signal Processing Conference (EUSIPCO), 2016, pp. 662666.

[10] PhysioBank ATM. [Online]. Available: https://physionet.org/cgi-bin/atm/ATM. [Accessed: 30-Jan2018].

[11] I. I. Christov, Real time electrocardiogram QRS detection using combined adaptive threshold, BioMedical Engineering OnLine, vol. 3, p. 28, 2004.

[12] Z. Zidelmal, A. Amirou, M. Adnane, and A. Belouchrani, QRS detection based on wavelet coefficients, Computer Methods and Programs in Biomedicine, vol. 107, no. 3, pp. 490496, Sep. 2012.

[13] S. A. Chouakri, F. Bereksi-Reguig, and A. Taleb-Ahmed, QRS complex detection based on multi wavelet packet decomposition, Applied Mathematics and Computation, vol. 217, no. 23, pp. 95089525, Aug. 2011.

\section{Acknowledgements}

The authors wish to thank the virtual FEDER hospital project of Lorraine, France (FEDER-Etat-Rgion Hpital Virtuel de Lorraine) for their valuable support.

Address for correspondence:

Pauline Guyot

CRAN Campus Science, Boulevard des Aiguillettes

Vandoeuvre-les-Nancy, France

Mail : pauline.guyot@univ-lorraine.fr 